\title{
Boron Enrichment and Phase Formation at TiN Twin Boundaries
}

\author{
C. Scheu, ${ }^{*}$ D. Hochauer, $* *$ J. Wagner, ${ }^{* *}$ V. Srot, ${ }^{* * *}$ K. Jurkschat, $* * * *$ N. Grobert, ${ }^{* * * *}$ and \\ C. Mitterer*****
}

* Department of Chemistry and Center for NanoScience (CeNS), Ludwig-Maximilians-University of Munich, D-81377 Munich, Germany

** Materials Center Leoben Forschung GmbH, A-8700 Leoben, Austria

*** Max-Planck-Institute for Metals Research, D-70569 Stuttgart, Germany

**** Department of Materials, Oxford University, Oxford OX1 3PH, UK

**** Department of Physical Metallurgy and Materials Testing, University of Leoben, A-8700 Leoben, Austria

TiN coatings play an important role in various applications. For example, they are used as protective coatings for machining tools, decorative items and implants due to their high hardness, good oxidation resistance, high wear resistance, chemical inertness, gold appearance, and biocompatibility. In a recent study, it was shown that the hardness of such coatings can be considerably improved by adding low amounts of B [1]. For instance, it was found that the hardness of a TiN coating containing 0.3 at. $\% \mathrm{~B}$ is $c a .30 \%$ higher compared to that of pure TiN [1]. X-ray diffraction experiments on this coating prepared by thermal chemical vapor deposition (CVD) revealed only a slight broadening of the peaks compared to the B free material but no additional peaks were detected [1]. Therefore, the aim of the present study was to indentify the location of the B atoms. For this we applied a combination of various transmission electron microscopy (TEM) techniques including high-resolution TEM (HRTEM), energy-filtering TEM (EFTEM), and electron energy-loss spectroscopy (EELS) in a scanning TEM (STEM).

HRTEM and EFTEM were performed at $300 \mathrm{keV}$ using the Oxford JEOL 3000F equipped with a Gatan GIF 2000. EELS and energy-loss near-edge structure (ELNES) investigations were conducted at $100 \mathrm{keV}$ on the Stuttgart VG HB501 UX STEM equipped with a Gatan UHV Enfina. The EELS/ELNES data were all acquired in spot mode (beam size $0.7 \mathrm{~nm}$ ) with a dispersion between 0.1 and $0.3 \mathrm{eV} /$ channel and a full width of half maximum (FWHM) of the zero-loss peak of $0.6 \mathrm{eV}$. The convergence and collection semi-angles were $10 \mathrm{mrad}$ and $6.5 \mathrm{mrad}$, respectively. EELS measurements were also carried out on the JEOL 3000F but are not included due to lower energy resolution.

TEM studies showed a columnar microstructure of the coating containing 0.3 at.\% B with a TiN grain size of $c a .200 \mathrm{~nm}$ (Fig. 1a). Planar defects were also observed which appeared as dark features in STEM high-angle annular dark field (HAADF) images, see Fig. 2a. EELS measurements were taken at these defects and within the TiN grains. Investigation of the energy-loss region of the B-K and $\mathrm{Cl}-\mathrm{L}_{2,3}$ edge revealed that $\mathrm{B}$ is only present at the defects while the TiN grains are B free (Fig. 2b). Some of the TiN grains contain concentrations of $\mathrm{Cl}$ impurities, originating from the $\mathrm{TiCl}_{4}$ precursor used in the CVD process. The B-K ELNES exhibits similar features to those reported for elemental $\mathrm{B}$ [2] or $\mathrm{TiB}_{2}$ (in random orientation) [3] and the N-K and Ti- $\mathrm{L}_{2,3}$ ELNES showed that they are similar for both the defects and grains. It is important to note that the N-K edge onset and peak position measured at the defect exclude the presence of BN. Using EFTEM we found that the B is homogenously distributed along the defects. 
HRTEM studies of the defects showed that the adjacent grains have a twin orientation relationship with the (111) planes of the fcc TiN parallel to the grain boundary (Fig. 1b, c). At some of these (111) twin boundaries a thin interfacial phase with a width of up to $1 \mathrm{~nm}$ is visible (Fig. 1b, c). The detected lattice spacing of this interfacial phase is $0.32 \mathrm{~nm}$ corresponding to the (0001) lattice plane spacing of $\mathrm{TiB}_{2}$. The hexagonal stacking sequence of the $\mathrm{TiB}_{2}$ can be easily achieved at the (111) twin boundary where locally a hexagonal stacking already exists. Most likely, the B atoms replace the $\mathrm{N}$ atoms at the (111) TiN twin boundary, however, ab-initio calculations are necessary to confirm this assumption. It is important to note that we were not able to detect B at any other grain boundary configuration.

References

[1] Wagner et al., Surf. Coat. Techn. 201 (2006) 4247.

[2] Gupper et al., Mh. Chem. 133 (2002) 837.

[3] Lie et al., Phys. Rev. B, 61 (2000) 1786.

[4] M. Kathrein is acknowledged for fruitful cooperation. Financial support from the European Union via the 0260019 ESTEEM project is gratefully acknowledged. NG gratefully acknowledges the Royal Society for their financial support.

a)

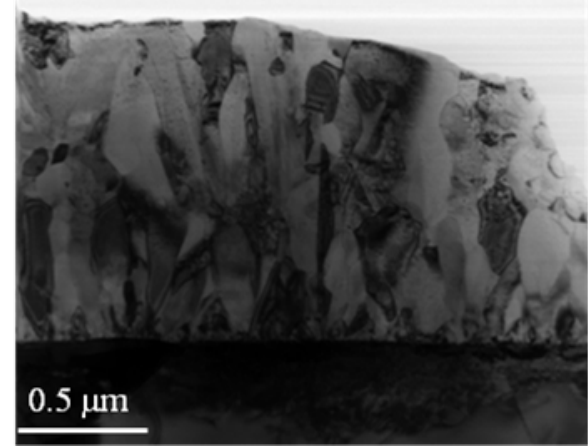

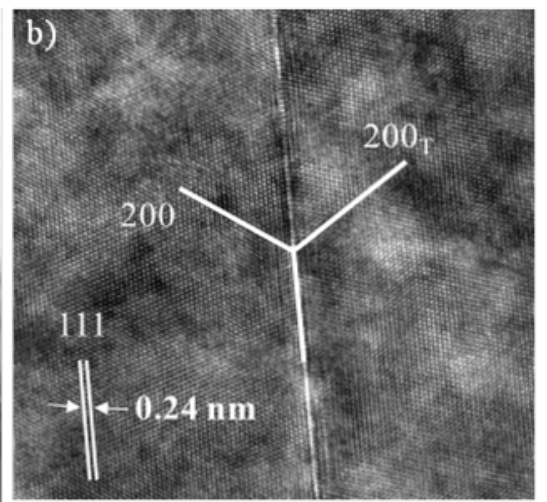

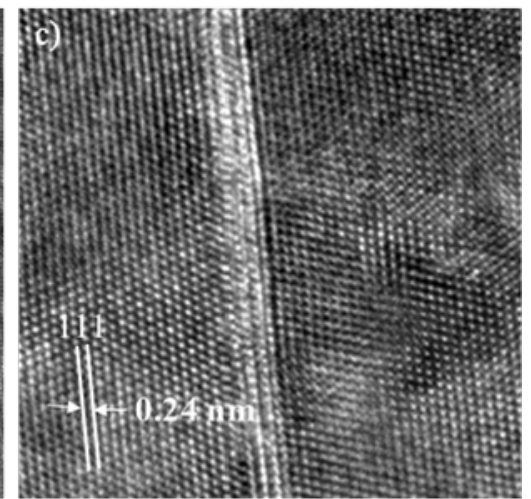

FIG. 1. Bright-field STEM image (a) showing the columnar microstructure. (b) and (c) are HRTEM images of the defects revealing a twin orientation relationship.
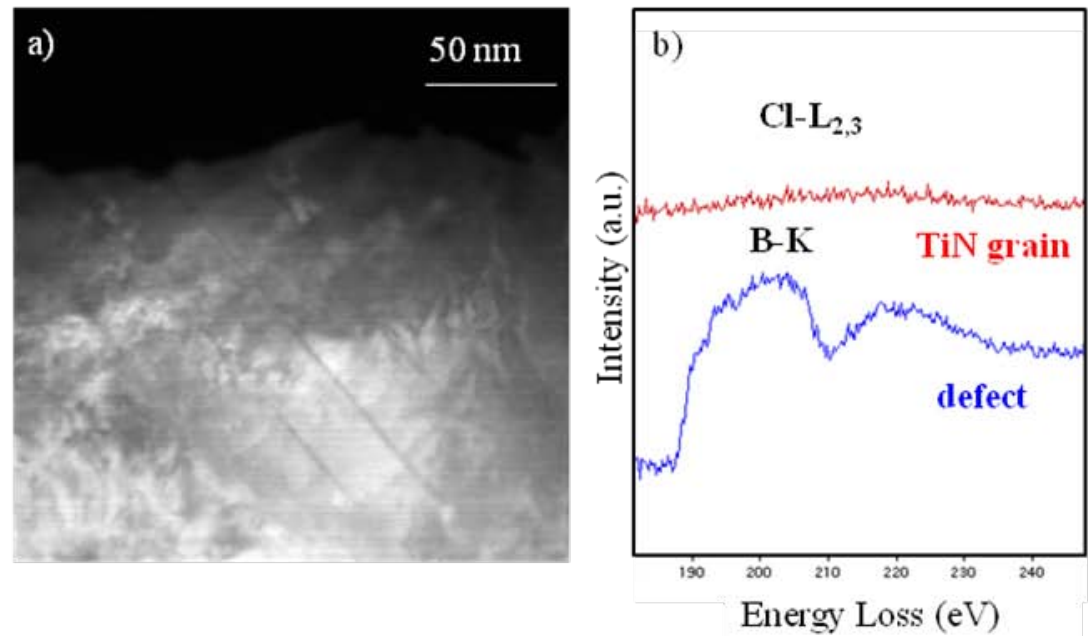

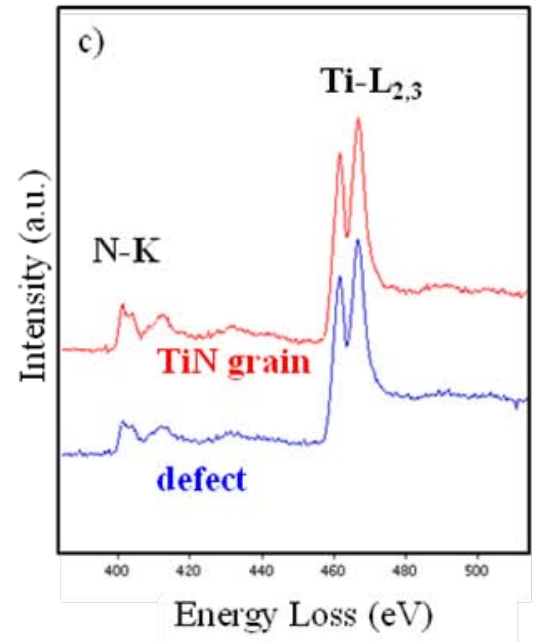

FIG. 2. HAADF image and corresponding EELS data taken at the middle defect. B is only located at the defects. 